\title{
POSTOPERATIVE SENSITIVITY AND CLINICAL EVALUATION OF POSTERIOR COMPOSITE RESTORATIONS IN MEDIUM AND DEEP CAVITIES PLACED USING TWO INSERTION TECHNIQUES (TWO-YEARS-RANDOMIZED CLINICAL STUDY)
}

\author{
Hala Ragab*
}

\begin{abstract}
Objective: This prospective randomized clinical study had two objectives, the first was to evaluate the influence of the cavity depth (medium and deep) and the insertion technique (flowable bulk and incremental layering) on the occurrence of postoperative sensitivity (POS) in singlesurface (class I) posterior composite resin restorations; and the second was to evaluate the clinical performance of these restorations over two-year period using selected united states public health service (USPHS) Criteria.
\end{abstract}

Methods: A total of 15-females and 17 males of an average age 25.6 \pm 6.1 years were enrolled in this clinical study. Patient selection was based on predetermined clinical criteria. In each patient, one pair of active occlusal caries (class I) of almost the same depth were included, each of which was located in a different quadrant (split-mouth design). Patients were divided into two groups based on the cavity depth: G1 included 21-pairs of medium-class I and G2 included 11-pairs of deep-class I. Cavities were randomly allocated to one of the two composite resin insertion methods: incremental layering using a nanohybrid resin composite only as control (CeramX-Mono) and a flowable bulk-fill base (SDR) veneered with CeramX-Mono. Methods used in cavity preparation, bonding procedures, finishing, and polishing were standardized and rubber-dam was used for all the clinical procedures. Evaluation of postoperative sensitivity (POS) was carried out at baseline (immediately after restoration), one-day, one-week, and one-month after treatment using cold/air stimuli, and recorded using a visual analog scale. The clinical evaluation of the restorations was carried out at baseline, one year and two-years, using modified USPHS selected relevant criteria according to van Dijken (1986): anatomic form, marginal integrity, color match and secondary caries. Scores of POS were statistically analyzed using Chi-square test, Fisher's exact test and ordinal regression at the level of significance $\mathrm{P} \leq 0.05$. Scores of the clinical criteria were analyzed using cumulative frequency distribution of scores. Fisher's Exact test was used to compare between the two cavity depths. Wilcoxon signed-rank test was used to compare between the two techniques. Friedman's test was used to study the changes by time within each group.

* Associate Professor, Department of Restorative Sciences, Faculty of Dentistry, Beirut Arab University, Beirut, Lebanon. 
Results: there was no statistically significant effect of gender and age on sensitivity grades. There was no significant difference between sensitivity grades at baseline for all groups. However, deep-cavity groups showed the significantly higher prevalence of mild to moderate sensitivity than medium-cavity groups. In a medium-cavity group, there was no statistically significant difference between sensitivity grades at one-day after restoration using the two techniques. All cases showed no POS at one-week and one-month. However, in the deep-cavity group, layering-technique showed the statistically significantly higher prevalence of mild sensitivity than bulk-fill technique one-day after restoration. There was no significant difference between the two techniques after one-week. Additionally, the entire deep-cavity group showed no sensitivity at one-month. Clinical evaluation of all restorations showed excellent performance at 2-year follow-up with $0 \%$ failure rate. The cavity depth and the insertion technique showed no significant effect on anatomical form, color match and marginal discoloration with a range of (scores 0-1). All cases showed excellent marginal continuity and no recurrent caries (score 0 ). However, in deep cavity groups at one-year evaluation, bulk fill showed a statistically significant higher prevalence of slight color mismatch (score 1) than layering technique which didn't change significantly after two-years.

Conclusion: Bulk fill technique based on SDR flowable base can be considered an alternative to conventional layering when the clinically relevant thickness is required. Bulk fill insertion may be significant in minimizing POS in high C-factor class I without sacrificing the physical and mechanical properties in stress-bearing areas. Both techniques showed acceptable clinical performance with predominantly excellent scores for all the clinical parameters analyzed in this 2-year follow-up clinical study.

\section{INTRODUCTION}

In modern dentistry, where conservation of tooth structure is of prime importance, resin-based composites have been widely used to restore teeth defects. Its indications have been extended to include many clinical situations including stressbearing areas (Rho et al 2013; Beck et al 2014). This was endorsed by the advances in material science, the vast improvement in the resin chemistry and adhesive systems and the continuous development of new materials and techniques that make the application of the material is more time-saving. The growing role of direct resin restoration indicates the requisite of following-up on their clinical performance.

Despite all the advances in the chemical formulation of resin composites, in the scientific literature, posterior composite restorations still experience some clinical problems. The inherent characteristics from resin composite such as polymerization shrinkage and elastic modulus of different dental structure can cause clinical manifestations, such as postoperative sensitivity, pulp inflammation, marginal defects, secondary caries and may also cause tooth or restoration fracture (Ritter 2008; Ferracane 2011). Moreover, any restorative material has a limited working lifespan. Longevity is affected by many factors other than the material properties, this includes patient and dentist related factors as well as the cavity type, location and geometry (van Dijken et al 2014).

Postoperative sensitivity (POS) is one of the main shortcomings of resin composites. Previous clinical research showed that up to $30 \%$ of the study populations have stated postoperative sensitivity after resin composite restoration placement (Briso et al 2007). The residual stresses resulted from volumetric polymerization shrinkage on curing was reported as the most serious issue with resin composites for most of the dental research (Ferracane JL, Hilton 2016). This becomes critical in deep cavities where the $\mathrm{C}$-factor is the highest as in case of class I restoration (Reis et al 2015). 
In order to reduce polymerization shrinkage, it was highly suggested to insert resin composites in layers of $2 \mathrm{~mm}$ increment taking into account the limited polymerization depth, furthermore to guarantee that one surface is bonded at once (Lynch et al 2014). Nevertheless, some clinical studies suggested that postoperative sensitivity has been still a problem for incremental resin composite restoration (Briso et al 2007).

Enhancements in material sciences have introduced the new era of "bulk-fill" composite restoratives that allow the composite restoration build-up in bulk, up to 4- $5 \mathrm{~mm}$ (Leprince et al 2014; Ayar 2016). Bulk-fill composites have some advantages over the conventional composites including the increased depth of cure, which possibly come from the greater translucency and low shrinkage stress which is related to modifications in the filler/resin matrix formulations (Lassila et al 2012; Leprince et al 2014). The first marketed lightcuring bulk-fill resin composite (SDR, Dentsply DeTrey; Konstanz, Germany), showed acceptable clinical results (Manhart et al 2009). Recently, several new materials have been marketed within this new class of bulk-fill resin composites and were divided into two groups with different mechanical properties, the low- and high-viscosity materials (Ilie et al 2013). Lower shrinkage stress was reported for the flowable material than for regular methacrylatebased resin composites and several nanohybrid flowable composites (Ilieand Hickel 2011). For SDR bulk-fill resin composite, polymerization stress was claimed to be reduced directly during curing. A patented urethane di-methacrylate polymerization modulator was chemically embedded in the resin backbone, which resulted in a slower modulus development, allowing stress reduction without decreasing the conversion rate (Alshali et al 2013; Ilie et al 2013a, 2013b; El-Damanhoury\& Platt 2014). Because of the favorable properties of SDR, it might be the ideal material to be used as dentin replacement that eliminates stresses at the interface, improve sealing ability, and prevent POS. However, when it comes to deep cavities with profound decay, it is not an easy decision to consider the use of bulkfill restorative as the standard of care. Clinical data regarding bulk-fill composite interactions with vital teeth with deep carious lesions seem to be very limited in the literature. Alike is the clinical studies investigating incremental resin composites and bulk-fill composites.

One clinical study evaluated the clinical effectiveness of the flowable bulk-fill composite technique in posterior restorations in comparison with incremental composite technique (van Dijken JW, Pallesen 2014). They reported that no significant difference in prevalence of postoperative sensitivity after restoration placement between groups. Another recent clinical study showed that postoperative sensitivity reported by patients after the restoration placement were not influenced by resin composite type (Ayar 2017). Although laboratory tests can provide relevant information on the restoration longevity, the long-term performance of restorative still depends on clinical evaluations. However, clinical studies require a more effective approach and greater precision in the experimental design to minimize the influence of other variables on the results. Since only a few clinical studies involving bulk-fill composites are available in the literature and up to our knowledge, none of them have specified the cavity depth, more detailed information regarding the clinical performance of bulk-fill in deep cavities would be desirable.

This prospective clinical study had two objectives, the first was to evaluate the influence of the cavity depth (medium and deep) and the insertion technique (flowable-bulk and incremental layering) on the occurrence of postoperative sensitivity (POS) in single-surface (class I) posterior composite resin restorations; and the second was to evaluate the clinical performance of these restorations over two-year period using selected united states public health service (USPHS) Criteria. 
The hypothesis tested was that there was no difference in POS and clinical performance between the medium and the deep class I resin composite restoration using the two insertion techniques over 2-years.

\section{MATERIALS AND METHODS:}

This prospective randomized controlled clinical study was conducted in the Specialty Clinic of Faculty of Dentistry at Beirut Arab University (BAU). After the approval of BAU -IRB (2016H-0035-D-R-0150), patients assigned for this study have signed an informed written consent form prior to participation in the clinical study. Patient records of individuals having at least a pair of class I primary active caries lesions in two different quadrants were enrolled in the study. Preoperative radiographs were taken. The cavity depth had been classified radiographically to be in outer, middle or inner one-third of the dentin (Unemori et al 2004). Only patient records which involve a pair of lesions in different quadrants either in the middle or inner dentin in the molar area were included in the study. The following parameters were set for sample size calculation: the expected proportion was 0.8 , the relative standard error was of $10 \%$, the power of the test was calculated to be $80 \%$ (beta error), and the two-tailed alpha error of $5 \%$ was 2 -sided. Based on these data, a sample size of 25 subjects was found to be necessary. Taking into consideration a possible loss during the study, a $20 \%$ increase in sample size was set totalizing 30 subjects (ADA 1972). A total of 32-patients who fulfilled specific clinical criteria have participated in this study (table 1).

\section{Cavity preparation and Grouping}

Caries was excavated by a spoon excavator and a conservative cavity design was made using tungsten carbide bur just to include the defective part. Remaining caries was removed until caries detector induced no further staining. Cavity depth was measured from the deepest area using the periodontal probe. Cavities were divided into two groups: cavities with scores 6 to 7 were allocated to G1 "medium cavity", and with scores 8 to 10 to G2 "deep cavity". For each patient, cavities were randomly allocated according to a predetermined scheme of randomization, to one of two insertion methods: incremental-layering using CeramXMono (control) on one quadrant and bulk-fill using SDR veneered with CeramX-Mono on the other quadrant.

\section{Restorative procedures}

All cavities were conditioned with $36 \%$ phosphoric acid, first the enamel margin then dentin walls for 15 seconds, rinsed and gently dried. Prime \& Bond ${ }^{\circledR}$ (Dentsply Detrey, Germany) was applied and light cured for 20 seconds. Incremental layering

Table 1: inclusion/exclusion criteria

\begin{tabular}{|l|l|}
\hline Inclusion criteria & Exclusion criteria \\
\hline $\begin{array}{l}\text { 1. Age between 18-30 years with good oral hygiene. } \\
\text { 2. Patients having active primary class I carious lesions on vital molar teeth } \\
\begin{array}{l}\text { in two-different quadrants and are opposed to natural dentition. } \\
\text { Teeth having a positive reaction to vitality test (cold test), no signs of pulp } \\
\text { inflammation, or spontaneous pain before treatment. }\end{array}\end{array}$ & $\begin{array}{l}\text { 1. Excessive tooth wear due to clenching or } \\
\text { abnormal habits. } \\
\text { tagonist cusp (traumatic occlusion). }\end{array}$ \\
$\begin{array}{l}\text { 4. Preoperative radiographic record of the carious lesions is either in the mid- } \\
\text { dle or the inner third of dentin in the two quadrants of each patient. } \\
\text { 5uccolingual width is no more than half the intercuspal distance. }\end{array}$ & $\begin{array}{l}\text { 4. Patients using analgesics and/or anti-in- } \\
\text { flammatory medicine. }\end{array}$ \\
\hline
\end{tabular}


was done by placing small horizontal increments while bulk-fill was done by placing $\mathrm{SDR}^{\mathrm{TM}}$ (Dentsply Detrey, Germany) in bulk up to $4 \mathrm{~mm}$ as needed to fill the cavity to the dento-enamel junction followed by a layer of $2 \mathrm{~mm}$ nanohybrid composite (CeramX-Mono, (Dentsply Detrey, Germany). No calcium hydroxide or other base material was used. Restorations were finished to the normal anatomy and polished using the Enhance ${ }^{\circledR}$ Finishing System and $\mathrm{PoGo}{ }^{\circledR}$ one-step diamond micro-polisher (Dentsply Detrey, Germany) and occlusion was adjusted.

The patients were blinded in regard to the material type of each quadrant and cavity depth. One operator did all restorative procedures under standardized conditions and rubber-dam isolation.

\section{Clinical Assessment}

Two examiners who did not participate in the restorative procedures and were unaware of the treatment carried out evaluated the restorations at all time of the study. The examiners used the Kappa index $(\mathrm{K}=0.901)$ to determine inter-examiner reliability.

\section{Evaluation of POS}

Patients were evaluated for POS using both, a 3-second air-blast at a pressure of 40-65psi and a cold stimulation using a cotton pellet and ethylene chloride at baseline, one day, one week, and one month after restoration. Measurements were recorded using visual analog scale (VAS). According to this scale, 0 meant no pain, 1-2 mild pain, 3 to 7 moderate pain, 8-9 severe pain, and 10 worst pains (Caselli \& Martins 2006).

Scores of the selected clinical criteria were analyzed using cumulative frequency distribution of scores. Fisher's Exact test was used to compare between the two cavity depths. Wilcoxon signedrank test was used to compare between the two techniques. Friedman's test was used to study the changes by time within each group. Statistical analysis was performed with IBM $^{\circledR}$ IBM Corporation, NY, USA.

TABLE (2) Modified USPHS for direct clinical evaluation

\begin{tabular}{|c|c|c|}
\hline \multirow[t]{2}{*}{ Category } & \multicolumn{2}{|l|}{ Score/criteria } \\
\hline & Acceptable & Unacceptable \\
\hline Anatomical form & $\begin{array}{l}0: \text { The restoration is contiguous with tooth anatomy } \\
\text { 1: Slightly under- or over-contoured restoration }\end{array}$ & $\begin{array}{l}\text { 2: Restoration is under contoured } \\
\text { 3: Restoration is missing partially or totally; } \\
\text { fracture of tooth structure }\end{array}$ \\
\hline Marginal integrity & $\begin{array}{l}0 \text { : excellent continuity, explorer doesn't catch } \\
\text { 1: slight explorer catches but doesn't penetrate crevice } \\
\text { 2: Crevice at margin, enamel exposed }\end{array}$ & $\begin{array}{l}\text { 3: obvious crevice at margin dentin maybe } \\
\text { exposed } \\
\text { 4: restoration mobile, fractured, or missing }\end{array}$ \\
\hline Color match & $\begin{array}{l}\text { 0: good color match } \\
\text { 1: slight mismatch }\end{array}$ & $\begin{array}{l}\text { 3: obvious mismatch } \\
\text { 4: gross mismatch }\end{array}$ \\
\hline $\begin{array}{l}\text { Marginal } \\
\text { discoloration }\end{array}$ & $\begin{array}{l}\text { 0: no discoloration evident } \\
\text { 1: slight staining can be polished }\end{array}$ & $\begin{array}{l}\text { 3: obvious staining can't be polished away } \\
\text { 4: gross staining }\end{array}$ \\
\hline Recurrent caries & $0:$ no caries & 1: secondary caries at restoration margin \\
\hline
\end{tabular}

${ }^{\circledR}$ SPSS, Inc., an IBM Company. 


\section{Clinical evaluation of restorations}

Selected relevant clinical parameters were evaluated at baseline (one week), at one year and at two year recalls as shown in the table (2). Results were collected, tabulated and statistically analyzed.

\section{RESULTS}

The present study was conducted on 32 subjects; 17 males and 15 females. The mean \pm standard deviation (SD) values of age were $25.6 \pm 6.1$ years with a minimum of 18.0 years and a maximum of 30 years. G1 included 21-patients with mediumcavities in two different quadrants whereas G2 included 11-subjects with deep-cavities. All patients were available at all periods of evaluations with no dropouts. There was no significant effect of age or gender. The Cohen's Kappa statistics (0.95) showed strong agreement between the examiners and no statistical difference was observed in their answers.

\section{POS Results:}

As regard to the cavity depth, there was a significant difference between G1 and G2 in POS records for both techniques at baseline. With bulkfill base, G2 showed statistically significantly higher prevalence of mild sensitivity $(45.5 \%)$ than G1 $(4.8 \%)$ at base line $(\mathrm{P}=0.011)$. No sensitivity was shown at 1 day, 1 week and 1 month (Table 3). With layering technique, G2 showed significantly higher prevalence of mild and moderate sensitivity $(54.5 \%, 9.1 \%)$ respectively than $\mathrm{G} 1(9.5 \%$ and $0 \%)$ respectively at baseline $(\mathrm{P}=0.005)$. There was no statistically significant difference between the two groups at 1 day and 1 week. No sensitivity was reported after 1 month (Table 3 ).

As regard to the insertion technique, $\mathrm{G} 1$ revealed no statistically significant difference between sensitivity grades after using the two techniques at all test time periods. All G1 experienced no

Table (3) Descriptive statistics and results of Chi-square (or Fisher's exact) tests for comparisons between sensitivity grades with medium and deep cavities

\begin{tabular}{|c|c|c|c|c|c|}
\hline Technique & Time & Sensitivity prevalence & Medium cavity (G1) & Deep cavity (G2) & P-value \\
\hline Bulk fill & Baseline & $\begin{array}{l}\text { No sensitivity } \\
\text { Mild sensitivity }\end{array}$ & $\begin{array}{l}20(95.2 \%) \\
1(4.8 \%)\end{array}$ & $\begin{array}{l}6(54.5 \%) \\
5(45.5 \%)\end{array}$ & $0.011 *$ \\
\hline \multirow{3}{*}{ Layering } & Baseline & $\begin{array}{l}\text { No sensitivity } \\
\text { Mild sensitivity } \\
\text { Moderate sensitivity }\end{array}$ & $\begin{array}{l}19(90.5 \%) \\
2(9.5 \%) \\
0(0.0)\end{array}$ & $\begin{array}{l}4(36.4 \%) \\
6(54.5 \%) \\
1(9.1 \%)\end{array}$ & $0.005^{*}$ \\
\hline & 1 day & $\begin{array}{l}\text { No sensitivity } \\
\text { Mild sensitivity } \\
\text { Moderate sensitivity }\end{array}$ & $\begin{array}{l}18(85.7 \%) \\
2(9.5 \%) \\
1(4.8 \%)\end{array}$ & $\begin{array}{l}7(63.6 \%) \\
4(36.4 \%) \\
0(0.0)\end{array}$ & 0.154 \\
\hline & 1 week & $\begin{array}{l}\text { No sensitivity } \\
\text { Mild sensitivity }\end{array}$ & $\begin{array}{l}21(100.0 \%) \\
0(0.0)\end{array}$ & $\begin{array}{l}10(90.9 \%) \\
1(9.1 \%)\end{array}$ & 0.344 \\
\hline
\end{tabular}

*: Significant at $P \leq 0.05$ 
sensitivity. G2 revealed no statistically significant difference between sensitivity grades after using the two techniques of insertion at baseline. While after 1 day, layering technique showed the significantly higher prevalence of mild sensitivity (36.4\%) than bulk-fill technique $(\mathrm{P}=0.045)$. After 1 week, there was no statistically significant difference between the two techniques. After 1 month, all cases with deep cavity showed no sensitivity (Table 4).

\section{Clinical Evaluation Results}

Clinical evaluation of all restorations showed excellent performance at 2-year follow-up with $0 \%$ failure rate. The cavity depth and the insertion technique showed no statistically significant effect on the anatomical form, the color match and the marginal discoloration with a range of (scores 0-1). All restorations showed excellent marginal continuity and no recurrent caries (score 0 ). However, at oneyear evaluation in deep cavity groups, restorations with bulk-fill base showed a statistically significant higher prevalence of slight color mismatch (score 1) than restorations with conventional layering, which didn't change significantly after two-years (table 5).

Based on Friedman's test, our results demonstrated no time-dependency. In all groups; there was no statistically significant change in the clinical scores at different time periods (table 6).

TABLE (4) Descriptive statistics of Chi-square tests for comparisons between sensitivity grades after using the two techniques

\begin{tabular}{|c|c|c|c|c|c|}
\hline Cavity depth & Time & Sensitivity prevalence & Bulk-fill & Layering & P-value \\
\hline \multirow[b]{2}{*}{ G1 (Medium) } & Baseline & $\begin{array}{l}\text { No sensitivity } \\
\text { Mild sensitivity }\end{array}$ & $\begin{array}{l}20(95.2 \%) \\
1(4.8 \%)\end{array}$ & $\begin{array}{l}19(90.5 \%) \\
2(9.5 \%)\end{array}$ & 1.000 \\
\hline & 1 day & $\begin{array}{l}\text { No sensitivity } \\
\text { Mild sensitivity } \\
\text { Moderate sensitivity }\end{array}$ & $\begin{array}{l}21(100.0 \%) \\
0(0.0) \\
0(0.0)\end{array}$ & $\begin{array}{l}18(85.7 \%) \\
2(9.5 \%) \\
1(4.8 \%)\end{array}$ & 0.199 \\
\hline \multirow[t]{3}{*}{ G2 (Deep) } & Baseline & $\begin{array}{l}\text { No sensitivity } \\
\text { Mild sensitivity } \\
\text { Moderate sensitivity }\end{array}$ & $\begin{array}{l}6(54.5 \%) \\
5(45.5 \%) \\
0(0.0)\end{array}$ & $\begin{array}{l}4(36.4 \%) \\
6(54.5 \%) \\
1(9.1 \%)\end{array}$ & 0.475 \\
\hline & 1 day & $\begin{array}{l}\text { No sensitivity } \\
\text { Mild sensitivity }\end{array}$ & $\begin{array}{l}11(100.0 \%) \\
0(0.0)\end{array}$ & $\begin{array}{l}7(63.6 \%) \\
4(36.4 \%)\end{array}$ & $0.045^{*}$ \\
\hline & 1 week & $\begin{array}{l}\text { No sensitivity } \\
\text { Mild sensitivity }\end{array}$ & $\begin{array}{l}11(100.0 \%) \\
0(0.0)\end{array}$ & $\begin{array}{l}10(90.9 \%) \\
1(9.1 \%)\end{array}$ & 1.000 \\
\hline
\end{tabular}

*: Significant at $P \leq 0.05$ 
TABLE (5) Descriptive statistics and results of Friedman's test for comparison between clinical criteria scores at different time periods (*Significant at $\mathrm{P} \leq 0.05, \mathrm{NC}+$ : Not Computed because the variable is constant)

\begin{tabular}{|c|c|c|c|c|c|c|c|c|}
\hline \multirow[b]{2}{*}{ Criteria } & \multirow{2}{*}{$\begin{array}{l}\text { Cavity } \\
\text { depth }\end{array}$} & \multirow[b]{2}{*}{ Time } & \multirow[b]{2}{*}{ Score } & \multicolumn{2}{|c|}{ Bulk fill } & \multicolumn{2}{|c|}{ Layering } & \multirow{2}{*}{$P$-value } \\
\hline & & & & $\mathrm{n}$ & $\%$ & $\mathrm{n}$ & $\%$ & \\
\hline \multirow{12}{*}{ 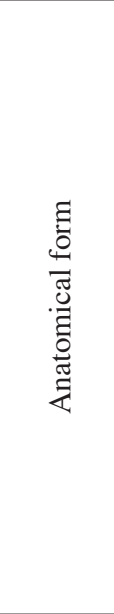 } & \multirow{6}{*}{ Medium } & \multirow{2}{*}{ Baseline } & Score 0 & $21 / 21$ & 100 & $21 / 21$ & 100 & \multirow{2}{*}{1.000} \\
\hline & & & Score 1 & $0 / 21$ & 0 & $0 / 21$ & 0 & \\
\hline & & \multirow{2}{*}{1 year } & Score 0 & $21 / 21$ & 100 & $21 / 21$ & 100 & \multirow{2}{*}{1.000} \\
\hline & & & Score 1 & $0 / 21$ & 0 & $0 / 21$ & 0 & \\
\hline & & \multirow{2}{*}{2 years } & Score 0 & $19 / 21$ & 90.5 & $20 / 21$ & 95.2 & \multirow{2}{*}{0.317} \\
\hline & & & Score 1 & $2 / 21$ & 9.5 & $1 / 21$ & 4.8 & \\
\hline & \multirow{6}{*}{ Deep } & \multirow{2}{*}{ Baseline } & Score 0 & $11 / 11$ & 100 & $11 / 11$ & 100 & \multirow{2}{*}{1.000} \\
\hline & & & Score 1 & $0 / 11$ & 0 & $0 / 11$ & 0 & \\
\hline & & \multirow{2}{*}{1 year } & Score 0 & $11 / 11$ & 100 & $11 / 11$ & 100 & \multirow{2}{*}{1.000} \\
\hline & & & Score 1 & $0 / 11$ & 0 & $0 / 11$ & 0 & \\
\hline & & \multirow{2}{*}{2 years } & Score 0 & $10 / 11$ & 90.9 & $11 / 11$ & 100 & \multirow{2}{*}{0.317} \\
\hline & & & Score 1 & $1 / 11$ & 9.1 & $0 / 11$ & 0 & \\
\hline \multirow{12}{*}{ 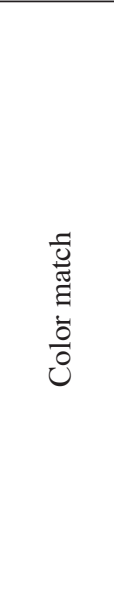 } & \multirow{6}{*}{ Medium } & \multirow{2}{*}{ Baseline } & Score 0 & $19 / 21$ & 90.5 & $21 / 21$ & 100 & \multirow{2}{*}{0.157} \\
\hline & & & Score 1 & $2 / 21$ & 9.5 & $0 / 21$ & 0 & \\
\hline & & 1 veor & Score 0 & $18 / 21$ & 85.7 & $21 / 21$ & 100 & 0002 \\
\hline & & 1 year & Score 1 & $3 / 21$ & 14.3 & $0 / 21$ & 0 & 0.083 \\
\hline & & 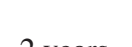 & Score 0 & $18 / 21$ & 85.7 & $19 / 21$ & 90.5 & 0217 \\
\hline & & 2 years & Score 1 & $3 / 21$ & 14.3 & $2 / 21$ & 9.5 & 0.317 \\
\hline & & Poscline & Score 0 & $8 / 11$ & 72.7 & $11 / 11$ & 100 & 0083 \\
\hline & & Baseline & Score 1 & $3 / 11$ & 27.3 & $0 / 11$ & 0 & 0.003 \\
\hline & $D_{2}$ & 1 & Score 0 & $6 / 11$ & 54.5 & $11 / 11$ & 100 & $002=*$ \\
\hline & Deep & 1 year & Score 1 & $5 / 11$ & 45.5 & $0 / 11$ & 0 & $0.025^{\circ}$ \\
\hline & & 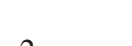 & Score 0 & $6 / 11$ & 54.5 & $9 / 11$ & 81.8 & ค0० \\
\hline & & 2 years & Score 1 & $5 / 11$ & 45.5 & $2 / 11$ & 18.2 & 0.005 \\
\hline & & D & Score 0 & $21 / 21$ & 100 & $21 / 21$ & 100 & 1000 \\
\hline & & Dasemine & Score 1 & $0 / 21$ & 0 & $0 / 21$ & 0 & 1.000 \\
\hline & Modinu & 1 yеar & Score 0 & $21 / 21$ & 100 & $21 / 21$ & 100 & 1000 \\
\hline.$\Xi$ & iviedintm & 1 year & Score 1 & $0 / 21$ & 0 & $0 / 21$ & 0 & 1.000 \\
\hline 䒕 & & 2 sorer & Score 0 & $21 / 21$ & 100 & $21 / 21$ & 100 & 1000 \\
\hline 范 & & 2 years & Score 1 & $0 / 21$ & 0 & $0 / 21$ & 0 & 1.000 \\
\hline $\bar{\Xi}$ & & Baseline & Score 0 & $11 / 11$ & 100 & $11 / 11$ & 100 & 1000 \\
\hline . & & & Score 1 & $0 / 11$ & 0 & $0 / 11$ & 0 & \\
\hline$\sum^{\pi}$ & Deen & 1 vear & Score 0 & $10 / 11$ & 90.9 & $11 / 11$ & 100 & 0317 \\
\hline & Deep & 1 yeal & Score 1 & $1 / 11$ & 9.1 & $0 / 11$ & 0 & 0.317 \\
\hline & & 2 yearc & Score 0 & $10 / 11$ & 90.9 & $10 / 11$ & 90.9 & 1000 \\
\hline & & 2 years & Score 1 & $1 / 11$ & 9.1 & $1 / 11$ & 9.1 & 1.000 \\
\hline
\end{tabular}


TABLE (6) Descriptive statistics and results of Friedman's test for comparison between clinical criteria scores at different time period ( $*$ Significant at $\mathrm{P} \leq 0.05, \mathrm{NC} \dagger$ : Not Computed because the variable is constant)

\begin{tabular}{|c|c|c|c|c|c|c|c|c|c|c|}
\hline \multirow{2}{*}{ Criteria } & \multirow{2}{*}{$\begin{array}{l}\text { Cavity } \\
\text { depth }\end{array}$} & \multirow{2}{*}{ Technique } & \multirow{2}{*}{ Score } & \multicolumn{2}{|c|}{ Base line } & \multicolumn{2}{|c|}{1 year } & \multicolumn{2}{|c|}{2 years } & \multirow[t]{2}{*}{$P$-value } \\
\hline & & & & $\mathrm{n}$ & $\%$ & $\mathrm{n}$ & $\%$ & $\mathrm{n}$ & $\%$ & \\
\hline \multirow{8}{*}{ 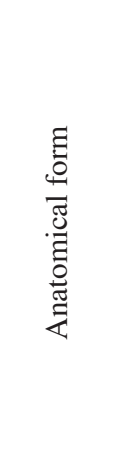 } & \multirow{4}{*}{ Medium } & \multirow{2}{*}{ Bulk-fill } & Score 0 & $21 / 21$ & 100 & $21 / 21$ & 100 & $19 / 21$ & 90.5 & \multirow{2}{*}{0.135} \\
\hline & & & Score 1 & $0 / 21$ & 0 & $0 / 21$ & 0 & $2 / 21$ & 9.5 & \\
\hline & & \multirow{2}{*}{ Layering } & Score 0 & $21 / 21$ & 100 & $21 / 21$ & 100 & $20 / 21$ & 95.2 & \multirow{2}{*}{0.368} \\
\hline & & & Score 1 & $0 / 21$ & 0 & $0 / 21$ & 0 & $1 / 21$ & 4.8 & \\
\hline & \multirow{4}{*}{ Deep } & \multirow{2}{*}{ Bulk fill } & Score 0 & $11 / 11$ & 100 & $11 / 11$ & 100 & $10 / 11$ & 90.9 & \multirow{2}{*}{0.368} \\
\hline & & & Score 1 & $0 / 11$ & 0 & $0 / 11$ & 0 & $1 / 11$ & 9.1 & \\
\hline & & \multirow{2}{*}{ Layering } & Score 0 & $11 / 11$ & 100 & $11 / 11$ & 100 & $11 / 11$ & 100 & \multirow{2}{*}{$\mathrm{NC}^{\dagger}$} \\
\hline & & & Score 1 & $0 / 11$ & 0 & $0 / 11$ & 0 & $0 / 11$ & 0 & \\
\hline \multirow{8}{*}{$\begin{array}{l}0 \\
\text { D } \\
\tilde{\Xi} \\
\tilde{0} \\
0 \\
\tilde{0} \\
0 \\
0\end{array}$} & \multirow{4}{*}{ Medium } & \multirow{2}{*}{ Bulk fill } & Score 0 & $19 / 21$ & 90.5 & $18 / 21$ & 85.7 & $18 / 21$ & 85.7 & \multirow{2}{*}{0.368} \\
\hline & & & Score 1 & $2 / 21$ & 9.5 & $3 / 21$ & 14.3 & $3 / 21$ & 14.3 & \\
\hline & & \multirow{2}{*}{ Layering } & Score 0 & $21 / 21$ & 100 & $21 / 21$ & 100 & $19 / 21$ & 90.5 & \multirow{2}{*}{0.135} \\
\hline & & & Score 1 & $0 / 21$ & 0 & $0 / 21$ & 0 & $2 / 21$ & 9.5 & \\
\hline & \multirow{4}{*}{ Deep } & \multirow{2}{*}{ Bulk fill } & Score 0 & $8 / 11$ & 72.7 & $6 / 11$ & 54.5 & $6 / 11$ & 54.5 & \multirow{2}{*}{0.135} \\
\hline & & & Score 1 & $3 / 11$ & 27.3 & $5 / 11$ & 45.5 & $5 / 11$ & 45.5 & \\
\hline & & \multirow{2}{*}{ Layering } & Score 0 & $11 / 11$ & 100 & $11 / 11$ & 100 & $9 / 11$ & 81.8 & \multirow{2}{*}{0.135} \\
\hline & & & Score 1 & $0 / 11$ & 0 & $0 / 11$ & 0 & $2 / 11$ & 18.2 & \\
\hline \multirow{8}{*}{ 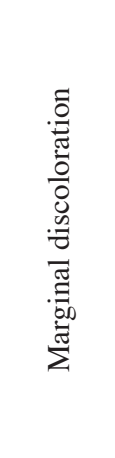 } & & & Score 0 & $21 / 21$ & 100 & $21 / 21$ & 100 & $21 / 21$ & 100 & \\
\hline & & 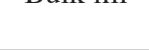 & Score 1 & $0 / 21$ & 0 & $0 / 21$ & 0 & $0 / 21$ & 0 & 10 \\
\hline & 19า & I avering & Score 0 & $21 / 21$ & 100 & $21 / 21$ & 100 & $21 / 21$ & 100 & \\
\hline & & & Score 1 & $0 / 21$ & 0 & $0 / 21$ & 0 & $0 / 21$ & 0 & \\
\hline & & & Score 0 & $11 / 11$ & 100 & $10 / 11$ & 90.9 & $10 / 11$ & 90.9 & \\
\hline & Deen & & Score 1 & $0 / 11$ & 0 & $1 / 11$ & 9.1 & $1 / 11$ & 9.1 & 0.500 \\
\hline & Dep & J ave & Score 0 & $11 / 11$ & 100 & $11 / 11$ & 100 & $10 / 11$ & 90.9 & 368 \\
\hline & & (2) & Score 1 & $0 / 11$ & 0 & $0 / 11$ & 0 & $1 / 11$ & 9.1 & 0.000 \\
\hline
\end{tabular}

\section{DISCUSSION}

Randomized clinical trials investigating bulk-fill composites are currently few in number and longterm clinical studies are the ultimate way to collect scientific evidence on the clinical effectiveness of restorative treatments. However, clinical studies demand a more effective approach and a greater precision in the experimental design due to several variables which can influence the results. These variables may include patient, operator, and material factors (Hickey et al 2016). This randomized controlled clinical study was carefully designed to avoid possible variability observed in several previous studies. The patient selection was 
limited to reliable and easily accessible individuals who exhibited a good standard of oral hygiene and a motivation to maintain good oral health. The age group was restricted to $18-30(25.6 \pm 6.1$ years $)$ to provide more harmonious groups in terms of the biology of the dentin-pulp organ. Additionally, younger patients are more sensitive to stimuli due to the large pulp and wider dentinal tubules thus more prone to develop POS. By age, the possibility of obturation of tubules and formation of irregular secondary/tertiary dentin is high which may affect the reaction to stimuli (Unemori et al 2001).

A split-mouth design was used with a pair of almost same cavity depth so that each patient act as his own control to facilitate reliable comparisons. The sample size was, therefore, consisted of relatively fewer participants than those using parallel group. In the literature, some reviews showed that molars have a higher risk of failure than premolars (Opdam et al 2014) whereas others showed no difference (Berk et al 2015). In this study, only molars were selected to avoid this conflict and to relatively standardize the cavity geometry. The cavity depth was evaluated radio-graphically before the intervention and measured after caries removal. All restorative treatment was conducted through a single professional to standardize the clinical procedures. Clinical assessment throughout the study was blinded using two external examiners who were not aware of the type of restoration so that the chance of bias was reduced.

In this study, the cavity depth had significantly influenced POS immediately after insertion in both bulk-fill and layering. Therefore, the first part of the null hypothesis was rejected. The higher prevalence of POS in deep cavities may be attributed to the fact that the remaining dentin thickness in deep cavities is relatively small compared to medium cavities. The dentinal tubules are at least two times wider $(2.5 \mu \mathrm{m})$ than that of med-dentin $(1.2 \mu \mathrm{m})$ (Owens $\&$ Johnson 2006). The short dentinal tubules in the small dentin thickness may provide less intra-tubular resistance to the hydrodynamic fluid flow (Pashley et al 2002). Also, no liner or other base material was used in deep cavities which may minimize the hydrodynamic fluid movement by promoting the formation of respiratory dentin in deep cavities (Costa et al 2003). All these factors may contribute to the increase the potential of dentin sensitivity.

It has been claimed that polymerization shrinkage can be decreased by the use of an incremental layering technique by placing the material in increments of $2 \mathrm{~mm}$ followed by light curing of each layer. The POS findings revealed no significant difference between the two insertion techniques in medium and deep cavities at baseline. The only significance was reported after one-day at which, the layering technique showed the significantly higher prevalence of mild sensitivity $(36.4 \%)$ than bulk-fill technique in the deep cavity group. Since the materials were distributed likewise in the same patient, the material variable is probably the main reason for this finding.

Resin composites with lower modulus of elasticity or slower curing rate may reduce the polymerization stress (Ilie et al 2013). For the flowable base SDR bulk-fill composite resin, polymerization stress is claimed to be reduced directly during curing due to the presence of patented polymerization modulator (urethane dimethacrylate), which resulted in a slower modulus development, allowing stress reduction without decreasing the conversion rate (Alshali et al 2013). This modulator is not present in the chemistry of the nanohybrid composite (CeramX-mono). Moreover, SDR is more translucent and can permit light more effectively allowing more conversion rate. Since complete polymerization reaction is achieved within 24 hours from curing (Alshali et al 2013), POS experienced in the second day of placement of nanohybrid composite maybe be attributed to the material composition and its lower degree of 
translucency (Frauscher KE, Ilie 2012) that might affect the degree of conversion and the length of the polymerization reaction specially in the deeper cavities, inducing stresses after one-day. It seems that layering technique could not eliminate these stresses as did the bulk-fill technique in deep cavities. This explanation is supported by a previous study that revealed the lowest shrinkage stress of SDR when compared to other flowable and non-flowable nanohybrid and microhybrid and a silorane-based resin composite (Ilie \& Hickel 2011). Moreover, an earlier finite element analysis concluded that the oblique layering technique produced the highest stresses (Versluis et al 1996; Abbas et al 2003).

Regardless of the depth of the cavity or the insertion technique, there was no significant difference in POS on day 7. The symptoms completely disappeared at one month. This could suggest that POS is a temporary symptom that resolves as the degree of conversion is increased by time bearing in mind that all the restorative steps aught be properly performed.

Excessive efforts have been made to develop low-shrinkage resin composites by changing filler amount, size, and shape, monomer structure or chemistry and by modifying the polymerization reaction (Ilie \& Hickel 2011). Previous in vitro studies revealed that several mechanical properties were similar for bulk-fill base and nanohybrid. The difference was related to lower hardness and modulus of elasticity of bulk-fill materials (Ilie et al 2013). The resin composite used in this study was a modified version of ormocer-based nanohybrid resin composite. The same material was used in both groups either with or without SDR flowable base. This may explain the comparable clinical performance over the two-year period. Using lower hardness and less rigid SDR base did not negatively influence the mechanical properties of the restoration. Utilizing a highly filled nanohybrid resin composite and achieving a high degree of polymerization maybe the key to obtaining superior physical and mechanical properties.

In the past few years, the majority of the randomized clinical studies on posterior restorations reported annual failure rate between $0.9 \%$ and $3.3 \%$. in which different microhybrid and nanohybrid RCs and adhesive systems were evaluated (van Dijken \& Pallesen 2013). Opdam et al. in 2014 stated in their systematic review a failure rate of $2.4 \%$ at 10 years and failure was associated with extensive large and deep cavities. The main shortcoming of the previous literature was that the depth and dimensions of the cavities were not specified (Van Ende 2018). The results of the present study revealed $0 \%$ annual failure rate during the 2-year follow-up along with clinically satisfactory performance, predominantly excellent scores (0-1) for all the parameters analyzed. No difference was observed between SDR-based restorations and the control nanohybrid resin composite-only restorations. The second part of the null hypothesis was therefore accepted.

The current study design was restricted to medium and deep class I cavities in a split mouth design under specific inclusion criteria, this may provide a more clinically relevant results. In accordance, a recent clinical study with up to 6-year follow-ups evaluated the same material of the present study and have situated the SDR flowable bulk-fill RC technique between the lower annual failure rate materials. They reported annual failure rate of $1.4 \%$ in class II and $0 \%$ in class I for the SDR restorations which was not significantly different from the the control nanohybrid resin compositeonly restorations (van Dijken\& Pallesen 2017).

Previous studies stated that wear and integrity of the adhesive interface may modify marginal adaptation during the first year period of clinical use. Despite the high C-factor, all restorations exhibited excellent marginal integrity over the period of the study which probably attributed to the integrity of the adhesive interface. In line to these results, excel- 
lent marginal integrity was reported by van Dijken\& Pallesen (2017) in a randomized, controlled 6-year evaluation using the same restorative materials but not the same adhesive system which was selfetch. In this study a total-etch adhesive was used. It seems that proper application steps of the adhesive system and restorative material is the key to successful and durable bonding interface. The only significant change in the evaluated clinical parameters was the slight color mismatch in deep cavities with flowable SDR base after one year. The thickness of SDR in deep cavities exceeded $4 \mathrm{~mm}$ and the additional SDR layer was cured separately to eliminate the possibility of lowering the conversion rate. The capping nanohybrid resin composite was $2 \mathrm{~mm}$ as per manufacturer instructions. Increasing the thickness of the highly translucent flowable base may be the reason behind the slight color mismatch. In deep cavities a thicker opaque hybrid resin composite maybe recommended to overcome the high translucency of the flowable base. Despite the excellent clinical performance over 2-years, it is recognized that longer follow-up period is necessary to confirm long-term stability of the restorations tested and to avoid overestimation of their clinical effectiveness.

\section{CONCLUSION}

Within the limitation of this study, bulk fill technique based on SDR flowable base can be considered an alternative to conventional layering with added benefits when the clinically relevant thickness is required in class I cavities. Bulk fill insertion may be significant in minimizing POS in high C-factor without sacrificing the physical and mechanical properties in stress-bearing areas. Both techniques showed acceptable clinical performance with predominantly excellent scores for all the clinical parameters analyzed in this 2-year followup clinical study. More clinical studies that focus on the proportions of restoration as in large and deep cavities are needed to explore the benefits and shortcomings of bulk fill insertion technique.

\section{REFERENCES}

- Abbas G, Fleming GJ, Harrington E, Shortall AC, Burke FJ. Cuspal movement and microleakage in premolar teeth restored with a packable composite cured in bulk or increments. J Dent 2003;31:437-444.

- Alshali RZ, Silikas N, Satterthwaite JD. Degree of conversion of bulk-fill compared to conventional resin-composites at two-time intervals. Dent Mater 2013;29:213-217.

- American Dental Association. Council on dental materials and devices. Recommended standard practices for clinical evaluation of dental materials and devices. J Am Dent Assoc 1972;84:388-90.

- Ayar MK. Postoperative sensitivity after placement of bulk-fill posterior restoration. J Res Med\&Dent Sci 2017;5(3):53-58.

- Ayar MK. Restoration of Posterior Cavities with BulkFill Composites: Case Reports. Int J Basic Clin Stud 2016;5:77-81.

- Beck F, Dumitrescu N, König F, Graf A, Bauer P, Sperr W. One-year evaluation of two hybrid composites placed in a randomized-controlled clinical trial. Dent Mater 2014;30:824-38.

- Beck F, Lettner S, Graf A, Vitriol B, Dumitrescu N, Bauer $\mathrm{P}$, et al. Survival of direct resin restorations in posterior teeth within a 19-year period (1996-2015): A meta-analysis of prospective studies. Dent Mater 2015;31(8):958-85.

- Briso ALF, Mestrener SR, Delicio G, Sundfeld RH, Bedran-Russo A, De Alexandre R, et al. Clinical assessment of postoperative sensitivity in posterior composite restorations. Oper Dent 2007;32:421-6.

- Caselli DSM, Martins LRM. Postoperative sensitivity in class I composite resin restorations in vivo. J Adhes Dent, 2006;8(1): 53-8.

- Costa CA, Giro EM, do Nascimento AB, Teixeira HM, Hebling J. Short-term evaluation of the pulpo-dentin complex response to a resin-modified glass-ionomer cement and a bonding agent applied in deep cavities. Dent Mater 2003;19:739-46.

- El-Damanhoury H, Platt J. Polymerization shrinkage stress kinetics and related properties of bulk-fill resin composites. Oper Dent 2014;39:374-382.

- $\quad$ Ferracane JL, Hilton TJ. Polymerization stress-Is it clinically meaningful? Dent Mater 2016;32:1-10.

- Ferracane JL. Resin composite - State of the art. Dent Mater 2011;27:29-38. 
- Frauscher KE, Ilie N. Depth of cure and mechanical properties of nano-hybrid resin-based composites with novel and conventional matrix formulation. Clin Oral Investig 2012;16:1425-34.

- Gianordoli-Neto R, Padovani GC, Mondelli J, de Lima Navarro MF, Mendonça JS, Santiago SL. Two-year clinical evaluation of resin composite in posterior teeth: A randomized controlled study. JCD. 2016;19(4):306.

- Hickey D, Sharif O, Janjua F, Brunton PA. Bulk dentine replacement versus incrementally placed resin composite: A randomized controlled clinical trial. J Dent. 2016; 46:18-22.

- Ilie N, Bucuta S, Draenert M. Bulk-fill resin-based composites: an in vitro assessment of their mechanical performance. Oper Dent 2013;38:618-625.

- Ilie N, Hickel R. Investigations on a methacrylate-based flowable composite based on the $\mathrm{SDR}^{\mathrm{TM}}$ technology. Dent Mater 2011;27:348-355.

- Ilie N, Hickel R. Resin composite restorative materials. Austr Dent J 2011;56:59-66.

- Ilie N, Keßler A, Durner J. Influence of various irradiation processes on the mechanical properties and polymerization kinetics of bulk-fil resin-based composites. J Dent 2013;41:695-702.

- Lassila LV, Nagas E, Vallittu PK, Garoushi S. Translucency of flowable bulk-filling composites of various thicknesses. Chin J Dent Res 2012; 15:31.

- Leprince JG, Palin WM, Vanacker J, Sabbagh J, Devaux J, Leloup G. Physico-mechanical characteristics of commercially available bulk-fill composites. J Dent 2014;42:9931000 .

- $\quad$ Lynch CD, Opdam NJ, Hickel R, Brunton PA, Gurgan S, Kakaboura A et al. Guidance on posterior resin composites: Academy of operative dentistry-European section. J Dent 2014;42:377-83.

- Manhart J, Chen H-Y, Hickel R. Clinical evaluation of the posterior composite QuiXfil in class I and II cavities: 4-year follow-up of a randomized controlled trial. J Adhes Dent 2009;12:1-7.

- Opdam NJ, van de Sande FH, Bronkhorst E, Cenci MS, Bottenberg P, Pallesen U, et al. Longevity of posterior composite restorations: a systematic review and metaanalysis. J DR 2014;93(10):943-9.

- Owens BM, Johnson WW. Effect of new generation surface sealants on the marginal permeability of class $\mathrm{V}$ resin composite restorations. Oper Dent, 2006; 31(4):481-8.

- Pashley EL, Agee KA, Pashley DH, Tay FR. Effects of one versus two applications of an unfilled, all-in-one adhesive on dentine bonding. J Dent, 2002;30(2-3): 83-90.
- Reis A, Loguercio AD, Schroeder M, Luque-Martinez I, Masterson D, Maia LC. Does the adhesive strategy influence the post-operative sensitivity in adult patients with posterior resin composite restorations: A systematic review and meta-analysis. Dent Mater 2015;31:1052-67.

- $\quad$ Rho YJ, Namgung C, Jin BH, Lim BS, Cho BH. Longevity of direct restorations in stress-bearing posterior cavities: A retrospective study. Oper Dent 2013;38:572-82.

- Ritter AV. Posterior composites revisited. J Esthet Restor Dent 2008;20:57-67.

- Unemori M, Matsuya Y, Akashi A, Goto Y, Akamine A. Composite resin restoration and postoperative sensitivity: clinical follow-up in an undergraduate program. J Dent 2001;29(1):7-13.

- Unemori M, Matsuya Y, Akashi A, Goto Y, Akamine A. Self-etching adhesives and postoperative sensitivity. Am J Dent 2004;17(3):191-5

- van Dijken JW, Pallesen U. A randomized controlled three-year evaluation of "bulk- filled" posterior resin restorations based on stress decreasing resin technology. Dent Mater 2014;30:245-51.

- van Dijken JW, Pallesen U. A six-year prospective randomized study of a nano-hybrid and a conventional hybrid resin composite in Class II res- torations. Dent Mater 2013;29:191-198.

- van Dijken JW, Pallesen U. A randomized 10-year prospective follow-up of Class II nanohybrid and conventional hybrid resin composite restorations. Journal of Adhesive Dentistry. 2014;16(6):585-92.

- van Dijken JWV, Pallesen U. Bulk-filled posterior resin restorations based on stress-decreasing resin technology: a randomized, controlled 6-year evaluation. Europ J Oral Invest 2017; 125(4):303-309.

- van Dijken JWV. A clinical evaluation of anterior conventional, microfiller and hybrid composite resin fillings. A six-year follow-up study. Acta Odont Scand 1986; 44:357-367.

- Van Ende, Annelies. "Bulk-Fill Composites." Dental Composite Materials for Direct Restorations. Springer, Cham 2018. 113-118.

- Van Ende A, De Munck J, Lise DP, Van Meerbeek B. BulkFill Composites: A Review of the Current Literature. J Adhes Dent 2017;1;19(2):95-109.

- Versluis A, Douglas WH, Cross M, Sakaguchi RL. Does an incremental filling technique reduce polymerization shrinkage stresses? J Dent Res 1996;75:871-878. 